\title{
Analysis of the Dynamic Characteristics of Elliptical Gears*
}

\author{
Xing LIU**, Kazuteru NAGAMURA*** and Kiyotaka IKEJO**** \\ ** Graduate School of Engineering, Hiroshima University \\ 1-4-1 Kagamiyama, Higashi-Hiroshima, 739-8527, Japan \\ E-mail: d091750@hiroshima-u.ac.jp \\ *** Department of Mechanical System Engineering, Hiroshima University \\ 1-4-1 Kagamiyama, Higashi-Hiroshima, 739-8527, Japan \\ E-mail: nagamura@mec.hiroshima-u.ac.jp \\ **** Department of Mechanical System Engineering, Hiroshima University \\ 1-4-1 Kagamiyama, Higashi-Hiroshima, 739-8527, Japan \\ E-mail: ikejo@mec.hiroshima-u.ac.jp
}

\begin{abstract}
To date, elliptical gear has been commonly used in automobile, automatic machinery, pumps, flow meters and printing presses for its particular non-uniform rotation. However, the dynamic characteristics of elliptical gears have not been clarified yet. In this study, The calculation as well as the experiment of two elliptical gears, which are a single elliptical gear and a double elliptical gear, is carried out to analyze the dynamic characteristics of elliptical gears. General factors including the torque, the rotation speed and the tooth root stress of the test gears are investigated. According to the analysis conducted in this study, the dynamic input torque variation of elliptical gear becomes larger along with the increase of operating gear rotation speed and the experimental one increases much faster than the calculated one over the Critical Rotation Speed of Tooth Separation (CRSTS) of elliptical gear. The experimental input rotation speed varies according to the variation of input torque, leading to the difference between the experimental output rotation speed and the desired one. The calculation results of the CRSTS of elliptical gears are almost equal to the experimental ones. The dynamic load variation ratios of elliptical gear at different angular position as well as their changing trends with operating gear rotation speed are quite different from each other. And the experimental dynamic load variation ratios of elliptical gear show difference from the calculated ones because of tooth separation and tooth impact. The agreement of the calculation and experimental results proves the validity of this study.
\end{abstract}

Key words: Gear, Elliptical Gear, Non Uniform Rotation, Torque Variation, Rotation Speed Variation, Tooth Separation, Dynamic Load

\section{Introduction}

Elliptical gear is a typical non-circular gear, which has an elliptical pitch curve and transmits a variable-ratio rotation and power simultaneously. Kinematically, an elliptical gear is equivalent to a crossed-link or a cam; however it has no connecting link and low friction loss, and that allows higher operating speeds and heavier loads. In addition, the gear characteristics such as compact size, accurate transmission and ease of dynamic balance are always preferable. Hence, elliptical gears are commonly used in automobile, automatic machinery, pumps, flow meters and printing presses ${ }^{(1)}$.

[DOl: 10.1299/jamdsm.6.484] 2012 (No. 12-0106)

Copyright (C) 2012 by JSME 
Elliptical gears have been studied for decades. For the application of elliptical gears, the design methods of them need to be figured out. A lot of effort has been taken on the

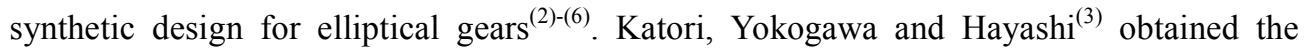
design process for the pitch curve of noncircular gears from various kinematic constraints, with given design specifications such as angular velocity ratio (AVR) curve and center distance. Figliolini and Angeles ${ }^{(5)(6)}$ formulated the synthesis of $N$-lobed elliptical gears with involute tooth profile by means of envelope theory and Euler-Savary Theorem. In particular, the formulation of both base curves for the right and left involute tooth profiles of $N$-lobed elliptical gears was obtained for the first time. On the kinematics of elliptical gears, Arakawa and Emura ${ }^{(7)}$ conducted the analysis of the contact ratio of noncircular gears. Three kinds of contact ratio (instantaneous contact ratio, pitch-average contact ratio and equivalent contact ratio) were proposed and their characteristics were discussed in this research. Then many studies focused on computer aided design and manufacture for elliptical gears ${ }^{(8)-(15)}$. Mathematical models of elliptical gears with not only involute teeth but also circular-arc teeth which were generated by existing equipments and tools including rack cutters, hob and shaper were developed. Tooth undercutting and pointed teeth were investigated in these researches. Particularly Litvin et al. ${ }^{(14)}$ proposed approaches to generate tooth surfaces by enveloping process for not only planar elliptical gears but also helical ones. Bair ${ }^{(15)}$ developed the tooth profile generation of a crowned elliptical gear, which prevented edge contact when an elliptical gear drive has axial misalignment. Some special applications of elliptical gears were also described in the literature ${ }^{(16)(17)}$. Emura and Arakawa $^{(16)}$ employed a noncircular gear (proven to be an elliptical gear) for a new steering mechanism, which had the capability of turning a carrier with a small radius. To obtain a grinding pattern preventing the lapping surfaces from repeatedly cutting the optical fibre end face in the same direction, Liu, Chang and Mundo ${ }^{(17)}$ designed an elliptical gear to drive one point of the coupler link along a Figure-8 pattern.

No research on the dynamic characteristics of elliptical gear has yet been described in the literature. Since elliptical gears conduct non-uniform rotation, the inertial torques are yielded when they rotate and increase along with the increase of operating speed, leading to the increase of transmitted load and unthought-of vibration. Therefore it is of great importance to know the dynamic characteristics of elliptical gears for engineers when designing them. In this study, the calculation as well as the experiment is carried out for two elliptical gears, which are a single elliptical gear and a double elliptical gear, to investigate the dynamic characteristics of elliptical gears. And for comparison, two circular gears with the same number of teeth, module, pressure angle, face width, center distance as the two elliptical gears respectively, are also investigated. The general factors including the dynamic torque, the dynamic rotation speed and the dynamic tooth root stress of the four test gears are measured by running tests. The torque variation ratios and the dynamic load variation ratios of the four test gears are calculated. Furthermore, the CRSTSs of elliptical gears which are critical parameter for the vibration analysis of elliptical gear are obtained.

\section{Motion expression}

\subsection{Gear properties of test gears}

In this study, two elliptical gear pairs, which are a single elliptical gear pair and a double elliptical gear pair, and two circular gear pairs, which are of the same number of teeth, module, face width, center distance as the two elliptical gear pairs respectively, have been tested. For convenience, the single elliptical gear and double elliptical gear are denoted as elliptical gear I and elliptical gear II. Correspondingly, the two circular gears are denoted as circular gear I and circular gear II.

Figure 1 shows the photos of the two elliptical gears. The gear properties of the four test 
gears are shown in Table 1.

\subsection{Motion expression of elliptical gears}

The motion of a crossed-link or a cam is usually depicted as the linear or angular displacement of the follower with respect to that of the driver. However, in this study, the AVR which is defined as the ratio of the angular velocity of the driven gear and the driving gear is employed to express the motion of elliptical gears. Let $\phi$ represent the angular displacement of the driven gear, $\theta$ be the angular displacement of the driving gear, $\varepsilon$ denote the flattening factor of the elliptical gears, $N$ depict the number of lobe of the elliptical gears, and $\varphi$ be the initial phase of the driving gear, then the AVR can be shown as the following equation $^{(18)}$ :

$$
\frac{d \phi}{d \theta}=\frac{\left(1-\varepsilon^{2}\right)}{\left(1+\varepsilon^{2}+2 \varepsilon \cos (N \theta+\varphi)\right)}
$$

The flattening factor of elliptical gear is defined as:

$$
\varepsilon=\frac{\left(r_{\max }-r_{\min }\right)}{\left(r_{\max }+r_{\min }\right)}
$$

where $r_{\max }$ and $r_{\min }$ are the maximum and minimum pitch curve radii of the elliptical gear respectively. For one-lobe elliptical gear, the flattening factor $\varepsilon$ is equal to the well known eccentricity of the ellipse $e$, calculated as

$$
e=\frac{\sqrt{a^{2}-b^{2}}}{a}
$$

where $a$ is the major radius of the ellipse and $b$ is the minor radius of the ellipse, while for

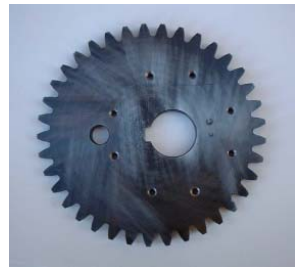

(a) Elliptical gear I

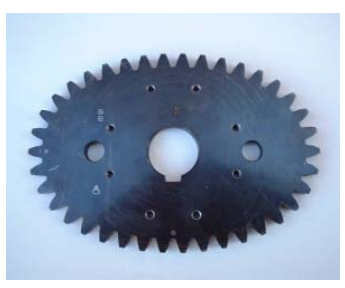

(b) Elliptical gear II

Fig. 1 Photos of elliptical gears

Table 1 Properties of test gears

\begin{tabular}{|c|c|c|c|c|}
\hline Gear type & $\begin{array}{c}\text { Elliptical } \\
\text { Gear I }\end{array}$ & $\begin{array}{c}\text { Circular } \\
\text { Gear I }\end{array}$ & $\begin{array}{c}\text { Elliptical } \\
\text { Gear II }\end{array}$ & $\begin{array}{c}\text { Circular } \\
\text { Gear II }\end{array}$ \\
\hline Module $m[\mathrm{~mm}]$ & \multicolumn{5}{|c|}{4.0} \\
\hline Pressure angle $\alpha_{0}\left[^{\circ}\right]$ & \multicolumn{5}{|c|}{20.0} \\
\hline Number of teeth $z_{1} / z_{2}$ & \multicolumn{2}{|c|}{$37 / 37$} & \multicolumn{2}{|c|}{$148 / 38$} \\
\hline Center distance $d[\mathrm{~mm}]$ & \multicolumn{2}{|c|}{149.51} & 0.2 & 0 \\
\hline Face width $b[\mathrm{~mm}]$ & \multicolumn{5}{|c|}{0} & -0.39 \\
\hline Flattening factor $\varepsilon$ & 0.2 & 0.14 & 1.713 & 1.959 \\
\hline Modification coefficient $x$ & - & 1.683 & 1.713 \\
\hline Contact ratio* $\varepsilon_{a}$ & 1.698 & \multicolumn{5}{|c|}{ S45C } \\
\hline Material & &
\end{tabular}

${ }^{*}$ For elliptical gears, it means average contact ratio ${ }^{(7)}$. 
two-lobe elliptical gear, $\varepsilon$ is not equal to $e$. The initial phase $\varphi$ represents the angular displacement when the driving gear starts to revolve from where the pitch curve radius is minimum. To simplify the formula, it is assumed that $\varphi$ is equal to zero in this study.

Once the AVR of an elliptical gear pair is determined, one can obtain the angular displacement of driven gear from integral of the AVR with respect to the angular displacement of driving gear, and the changing rate of the AVR, named as angular acceleration ratio (AAR) and denoted as $d^{2} \phi / d \theta^{2}$, from the derivative of the AVR with respect to the angular displacement of driving gear. The calculation formulae of the angular displacement of driven gear and AAR are shown as Eqs. (4) and (5) respectively:

$$
\begin{gathered}
\phi=\frac{2}{N} \arctan \left(\frac{1-\varepsilon}{1+\varepsilon} \tan \frac{N \theta}{2}\right)+\mathrm{C} \\
\frac{d^{2} \phi}{d \theta^{2}}=\frac{2 N \varepsilon\left(1-\varepsilon^{2}\right) \sin (N \theta)}{\left(1+\varepsilon^{2}+2 \varepsilon \cos (N \theta)\right)^{2}}
\end{gathered}
$$

Figure 2 shows the motion curves of elliptical gear I and elliptical gear II.

\section{Experimental apparatus and method}

\subsection{Experimental apparatus}

In this study, a power absorption type gear testing machine is used for dynamic experiments. Figure 3 shows the scheme of this experimental apparatus, which consists of a variable-speed three-phase asynchronous motor, two torque meters, two rotary encoders or slip-rings, a test gear pair, and an electromagnetic powder brake. The dynamic tooth root stress is measured by using the strain gauge stuck on the non-mesh-side tooth root of the driven gear as shown in Fig. 4.

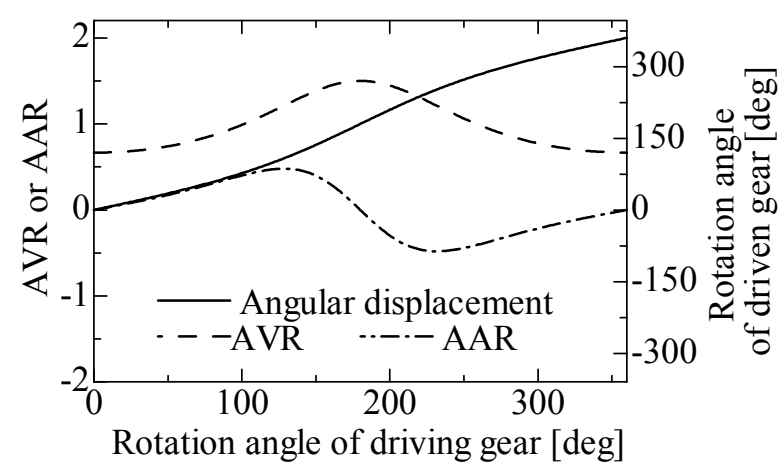

(a) Elliptical gear I $(N=1, \varepsilon=e=0.2)$;

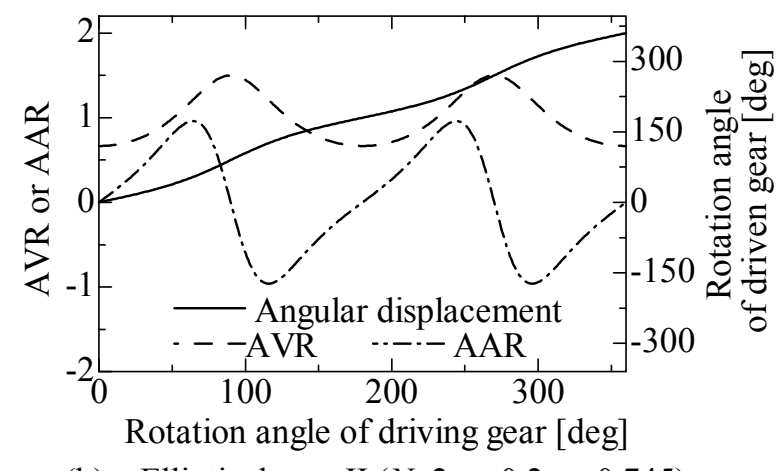

(b) Elliptical gear II ( $N=2, \varepsilon=0.2, e=0.745)$.

Fig. 2 Motion curves of elliptical gears 


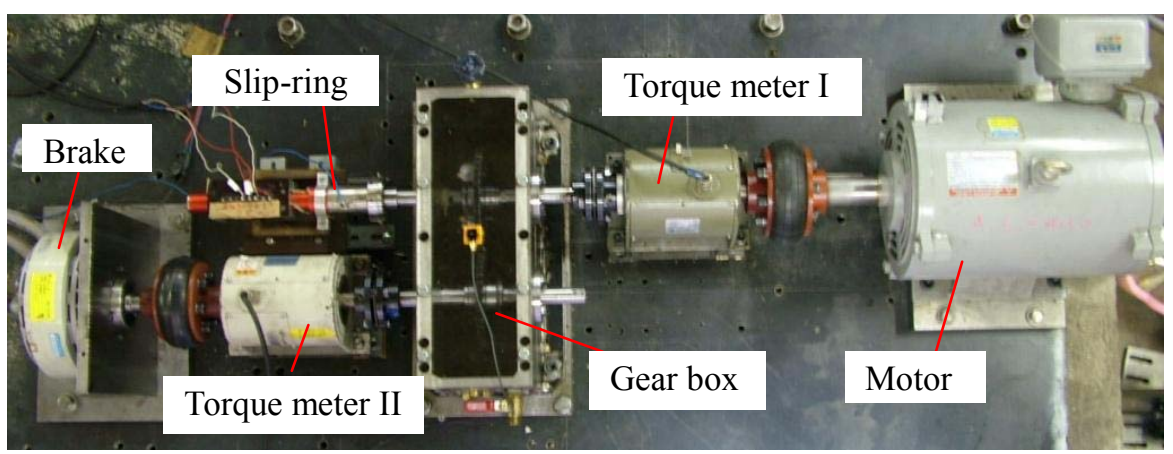

Fig. 3 Scheme of the power absorption type gear testing machine

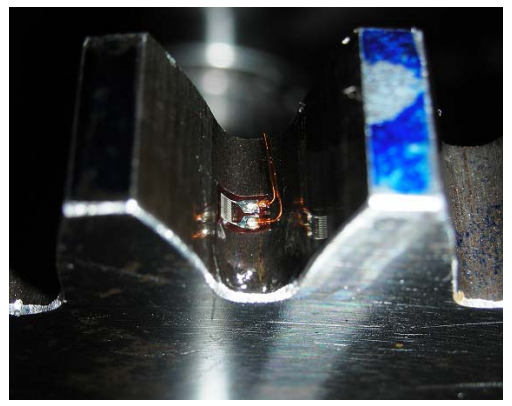

Fig. 4 Position of the strain gauge stuck for measuring tooth root stress

\subsection{Experimental method}

Both the dynamic input and output torques, both the dynamic input and output rotation speeds and the dynamic tooth root stress of the non-mesh-side of driven gear of the four test gears are measured by running test. Load torques are set for $9.8 \mathrm{~N}-\mathrm{m}$ and $19.6 \mathrm{~N}-\mathrm{m}$ by the electromagnetic powder brake. The apparatus is driven by the three-phase asynchronous motor for various operating gear rotation speeds. For elliptical gear I and circular gear I, the operating gear rotation speeds are set from $120 \mathrm{rpm}$ to $840 \mathrm{rpm}$, while, for elliptical gear II and circular gear II, the operating gear rotation speeds are set from $120 \mathrm{rpm}$ to $540 \mathrm{rpm}$, with each increasing by $60 \mathrm{rpm}$.

\section{Calculation method and results}

In this section, the dynamic calculation is carried out to analyze the dynamic characteristics of elliptical gears. However, the calculation for the dynamic characteristics of elliptical gears in this study is a simplified one based on the following assumptions:

1. All the parts of the experimental apparatus are considered as rigid bodies. There is no dynamic vibration between the parts of the experimental apparatus.

2. There is no gear backlash between meshing gear pair. When the meshing side of gear pair converts between the mesh-side and the non-mesh-side of the gear pair, no tooth impact occurs.

3. The rotation speed of the driving side of the experimental apparatus keeps constant when operating, and then the rotation speed of the driven side accords with the AVR curve of the elliptical gear shown in Fig. 2 and is the desired output rotation speed. Therefore, the inertial torque of the driven side is yielded from the motion variation of the driven side.

4. Along with the increase of operating gear rotation speed, the inertial torque of elliptical gear becomes large, leading to the appearance of tooth separation and 
non-mesh-side mesh. When tooth separation occurs the input torque becomes zero and when non-mesh-side mesh occurs the input torque becomes negative.

For clear comparison with experimental results, some calculation results are shown together with the experimental results in Section 5.

\subsection{Calculation of torque}

The input and output torques of the experimental apparatus with elliptical gears can be calculated from the AVR and AAR of elliptical gear.

In the calculation based on the assumptions in this study, the rotation speed of the driving side, i.e. the input rotation speed is considered constant and equal to the motor speed, presented as $\omega_{i n}$. Then the driven side of the experimental apparatus conducts variable-speed rotation according to the AVR of elliptical gears. This rotation yields inertial torque and asks for input torque from the driving side just like load torque. Let $T_{i \text {-driven }}$ present the total inertial torque of the driven side, $J_{\text {driven }}$ depict the total moment of inertia of driven side and the inertial torque of driven side could be calculated as:

$$
T_{i-d r i v e n}=J_{\text {driven }} \omega_{i n}^{2} \frac{d^{2} \phi}{d \theta^{2}}
$$

Then the input torque could be calculated as:

$$
T_{\text {in }}=\left(T_{l}+T_{i-\text { driven }}\right) \frac{d \phi}{d \theta}
$$

where $T_{i n}$ is the input torque, $T_{l}$ is the load torque which is loaded by the electromagnetic powder brake in this study.

The output torque of elliptical gear also changes due to the inertial torque of the parts following the output torque meter (torque meter II shown in Fig. 3). Similar to the calculation of the total inertial torque of driven side, the inertial torque of the following parts of output torque meter, denoted as $T_{i-o u t}$, can be calculated as:

$$
T_{i-o u t}=J_{\text {out }} \omega_{\text {in }}^{2} \frac{d^{2} \phi}{d \theta^{2}}
$$

where $J_{\text {out }}$ is the total moment of inertia of the following parts of output torque meter.

Then the output torque $T_{\text {out }}$ could be calculated as:

$$
T_{\text {out }}=T_{l}+T_{\text {i-out }}
$$

\subsection{Calculation of rotation speed}

The input rotation speed of elliptical gear $\omega_{\text {in }}$ is considered constant and equal to the motor speed in this study. Then the rotation speed of the driven side, i.e. the output rotation speed of elliptical gear which is denoted as $\omega_{\text {out }}$ can be calculated according to the AVR of elliptical gear as the following formula:

$$
\omega_{\text {out }}=\omega_{\text {in }} \frac{d \phi}{d \theta}
$$

\subsection{Calculation of the CRSTS of elliptical gear}

The Critical Rotation Speed of Tooth Separation (CRSTS) of elliptical gear is an important index for the vibration analysis of elliptical gears. If the operating gear rotation speed goes over the CRSTS of elliptical gear, tooth separation will occur and then non-mesh-side tooth impact and mesh-side tooth impact will also happen. Upon that, the vibration of elliptical gear will become very much large, which will lead to the increase of transmitted load and cause unthought-of problems.

When tooth separation occurs, there is no torque transmitted between the gear pair and 
the torque of input shaft becomes zero. Set the value of $T_{\text {in }}$ in Eq. (7) as zero and then the CRSTS of elliptical gear can be derived. The calculation formula of the CRSTS of elliptical gear is shown as:

$$
\omega_{C R S T S}=\sqrt{\frac{-T_{l}}{\left.J_{\text {driven }} \frac{d^{2} \phi}{d \theta^{2}}\right|_{\min }}}
$$

\subsection{Calculation of tooth root stress}

In this study, the dynamic load is investigated according to the dynamic tooth root stress (the maximum dynamic tooth root stress during the tooth mesh circle is taken into account). Based on the assumptions of calculation in this study, the calculated tooth root stress of circular gear is constant, showing no increase neither along with the increase of operating gear rotation speed nor with the angular position of driving gear. However, the calculated tooth root stress of elliptical gear varies along with the angular position of driving gear, as well as along with the increase of operating gear rotation speed, due to the variation of AVR and the pitch curve of elliptical gear.

Figure 5 shows some teeth at different angular position of elliptical gears, marked as number 1, 14, 19 and 24 for the driven gear of elliptical gear I and number 1, 5, 11 and 16 for the driven gear of elliptical gear II. Recalling Fig. 2 in section 2, the teeth marked as number 1, 14, 19 and 24 of elliptical gear I correspond to the rotation angle of driving gear $0^{\circ}, 128^{\circ}, 180^{\circ}$ and $232^{\circ}$ respectively, while the teeth marked as number $1,5,11$ and 16 of elliptical gear II correspond to the rotation angle of driving gear $0^{\circ}, 64^{\circ}, 90^{\circ}$ and $116^{\circ}$ respectively, which are respectively the teeth with the minimum AVR (AAR is zero), maximum AAR, maximum AVR (AAR is zero) and minimum AAR of elliptical gear I or elliptical gear II. These four specific teeth of elliptical gear represent the four specific types of tooth load conditions and can be used to guess the load condition of other teeth.

The transmitted force of elliptical gear pair can be calculated from the division of the calculated input torque of the gear pair and the base curve radius of the driving gear. Let $r_{\mathrm{b} \theta}$ present the base curve radius of the driving gear and $r_{\mathrm{b} \theta}$ can be calculated as:

$$
r_{b \theta}=\frac{d \cos \alpha_{o}\left(1-\varepsilon^{2}\right)}{2(1+\varepsilon \cos (N \theta))}
$$

Then the transmitted force of elliptical gear pair, denoted as $W_{c a l}$, can be calculated as the following formula:

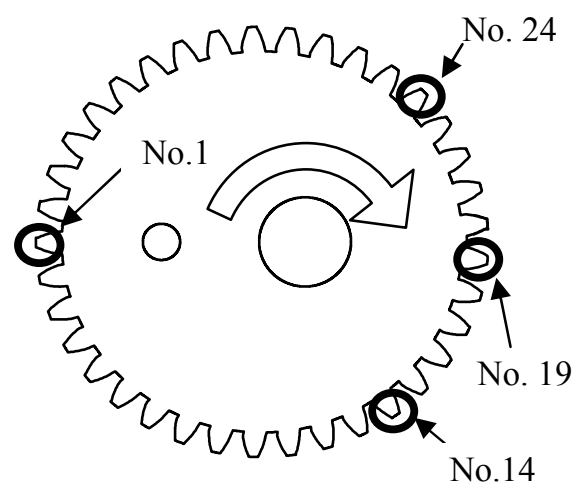

(a) Elliptical gear I

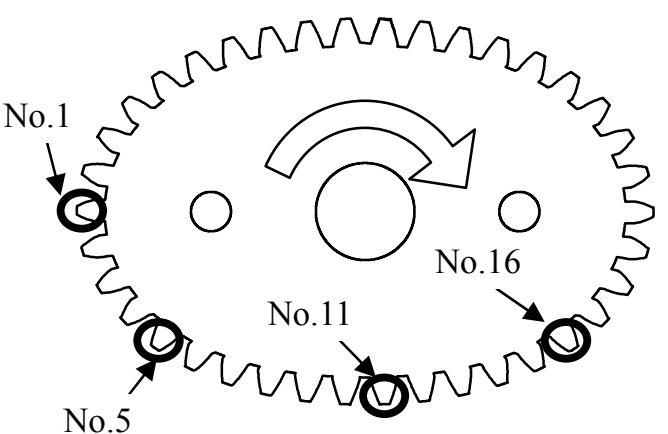

(b) Elliptical gear II

Fig. 5 Marked tooth numbers of the driven gear of elliptical gears 


$$
W_{c a l}=\frac{T_{i n}}{r_{b \theta}}
$$

In this study, Aida-Terauchi's Formula ${ }^{(19)}$ is used to calculate the tooth root stress:

$$
\sigma_{c a l}=A \frac{W_{c a l}}{b}
$$

where $\sigma_{c a l}$ is the calculated tooth root stress of the non-mesh-side of driven gear, $b$ is the gear face width, $A$ is the tooth root stress influence coefficient, which is obtained by Aida and Terauchi in Ref. (19) and varies along with the tooth meshing position.

By Eq. (14), the tooth root stress at different angular position of elliptical gears is calculated. The calculated dynamic load variation ratio of elliptical gear, which is defined as the ratio of the calculated tooth root stress of elliptical gear at operating gear rotation speeds to that of corresponding circular gear, is used to describe the calculated dynamic load variation of elliptical gear with respect to gear rotation speed in this study.

Figure 6 shows the calculated dynamic load variation ratios at different angular position of elliptical gears versus gear rotation speed for load torque $9.8 \mathrm{~N}-\mathrm{m}$. As shown in Fig. 6, the calculated dynamic load at different tooth of elliptical gear as well as its varying trend with the gear rotation speed is different from each other. Due to the inertial torque resulting from the non-uniform rotation of elliptical gear, the calculated dynamic load variation ratios of tooth No.14 of elliptical gear I and tooth No.5 of elliptical gear II increase along with the gear rotation speed and rise up respectively to 11 at $840 \mathrm{rpm}$ and to 10 at $540 \mathrm{rpm}$; contrarily, the calculated dynamic load variation ratios of tooth No.24 of elliptical gear I and tooth No.16 of elliptical gear II decrease and become negative, down to -8 at $840 \mathrm{rpm}$ and -7 at $540 \mathrm{rpm}$ respectively. The calculated dynamic loads of tooth No.1 and tooth No.19 of elliptical gear I and tooth No.1 and tooth No.11 of elliptical gear II keep constant with respect to gear rotation speed, although the calculated dynamic load of tooth No. 19 of elliptical gear I is a little larger than that of tooth No.1 of elliptical gear I and the calculated dynamic load of tooth No.11 of elliptical gear II is a little larger than that of tooth No.1 of elliptical gear II respectively.

Under the same gear rotation speed, the calculated dynamic load variation ratio of tooth No.5 of elliptical gear II is larger than that of tooth No.14 of elliptical gear I, and the calculated dynamic load variation ratio of tooth No.16 of elliptical gear II is smaller than that of tooth No.24 of elliptical gear I. These are considered as the inertial torque of elliptical gear II is larger than that of elliptical gear I under the same gear rotation speed.

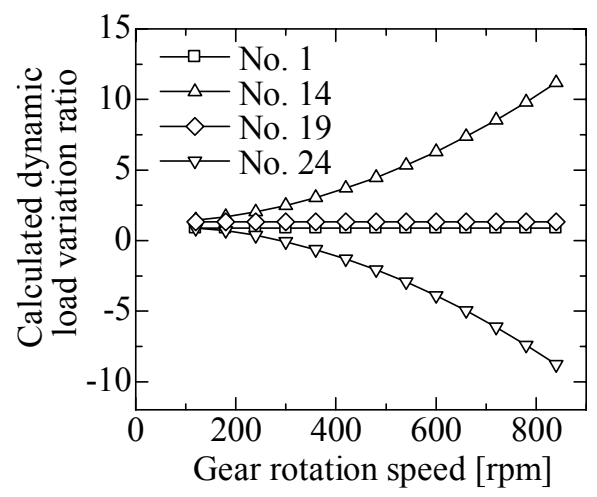

(a) Elliptical gear I

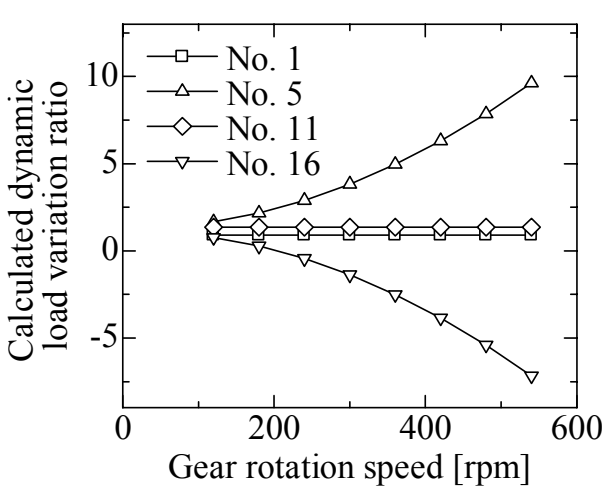

(b) Elliptical gear II

Fig. 6 Calculated dynamic load variation ratios of elliptical gears for $T_{l}=9.8 \mathrm{~N}-\mathrm{m}$ 


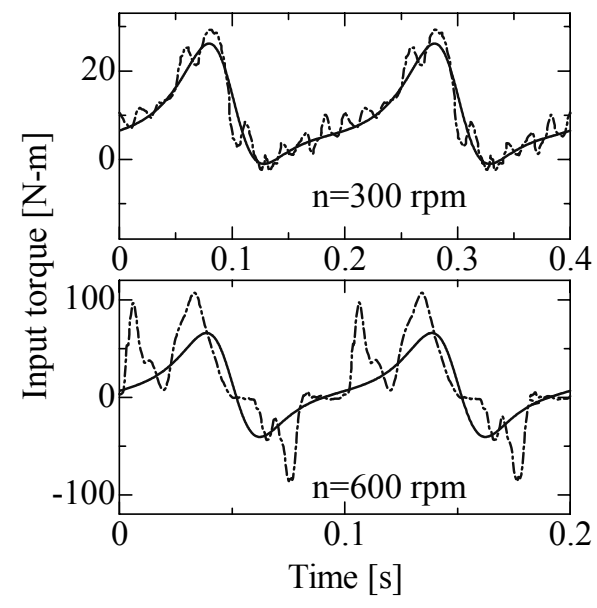

(a) Input torque

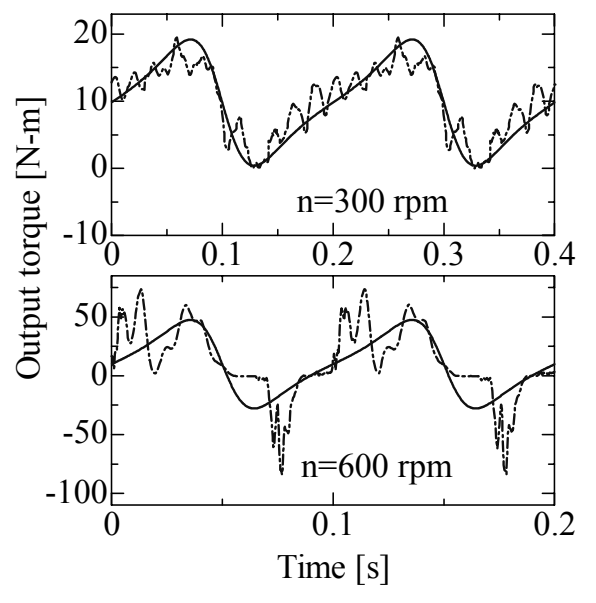

(b) Output torque

Fig. 7 Calculation and experimental results of input and output torque variations for elliptical gear $\mathrm{I}, T_{l}=9.8 \mathrm{~N}-\mathrm{m}$

------ Experimental input or output torque _-Calculated input or output torque

\section{Experimental results}

\subsection{Experimental results of torque}

Figure 7 shows the calculation and experimental results of both the input and output torque variation waveforms of elliptical gear I during 2 gear revolutions for load torque 9.8 $\mathrm{N}-\mathrm{m}$. The instantaneous AVRs of each time point of the calculated and experimental torque variation waveforms are made consistent to make sure that the calculated and experimental torque variation waveforms are at the same time.

As shown in Fig. 7, the trends of the calculated and experimental input and output torque variation waveforms agree with each other. The experimental torque variation waveforms show dynamic vibration and, therefore, show difference from the calculated ones. As a result of the inertial torque yielded from the non-uniform rotation, both the calculated and experimental torque variations of elliptical gear get large along with the increase of operating gear rotation speed. The torque variation frequency of elliptical gear agrees with the motion variation frequency of elliptical gear. Under higher operating gear rotation speeds, the experimental torque variation waveforms show flat zones and negative values. These are considered as the appearance of tooth separation and non-mesh-side tooth impact under the operating gear rotation speed higher than the CRSTS of elliptical gear. Along with the increase of operating gear rotation speed, the dynamic vibration of the experimental torque variation waveform becomes larger. This is because tooth separation and tooth impact occur and become acuter along with the operating gear rotation speed.

Input torque indicates the required torque from the motor for the apparatus. In this study, the input torque variation ratio which is defined as the ratio of the amplitude input torque value to the average input torque value (the load torque in this study) is calculated and employed to describe the input torque variation of elliptical gear. Figure 8 shows the calculation and experimental results of the input torque variation ratios versus the operating gear rotation speeds for the four test gears for load torque $9.8 \mathrm{~N}-\mathrm{m}$ and $19.6 \mathrm{~N}-\mathrm{m}$. As shown in Fig. 8, both the calculated and experimental input torque variation ratios of elliptical gears are much larger than those of circular gears and the experimental ones increase rapidly over a certain gear rotation speed, which is considered to be around the CRSTS of elliptical gear. Both the calculated and experimental input torque variation ratios of elliptical gear II are larger than elliptical gear I because of the larger inertial torque of 
elliptical gear II. Under operating gear rotation speeds lower than the CRSTS of elliptical gear, the torque variation ratios of experimental input torque are a little larger than the calculated ones. However, under higher operating gear rotation speeds, the torque variation ratios of experimental input torque get much larger than calculated ones, about 2 times large for elliptical gear I at $840 \mathrm{rpm}$ under $9.8 \mathrm{~N}-\mathrm{m}$ and 2.5 times large for elliptical gear II at 540 rpm under $9.8 \mathrm{~N}-\mathrm{m}$. These are considered as the dynamic vibration gets large under higher operating gear rotation speed because of tooth impact. For load torque $19.6 \mathrm{~N}-\mathrm{m}$, the torque variation ratios are accordantly lower than the ones for load torque is $9.8 \mathrm{~N}-\mathrm{m}$.

\subsection{Experimental results of rotation speed}

Figure 9 shows the comparison of the calculation and experimental results of both the input and output rotation speed variation waveforms of elliptical gear I during 3 revolutions for load torque $9.8 \mathrm{~N}-\mathrm{m}$. The instantaneous rotation speeds of input and output shaft are

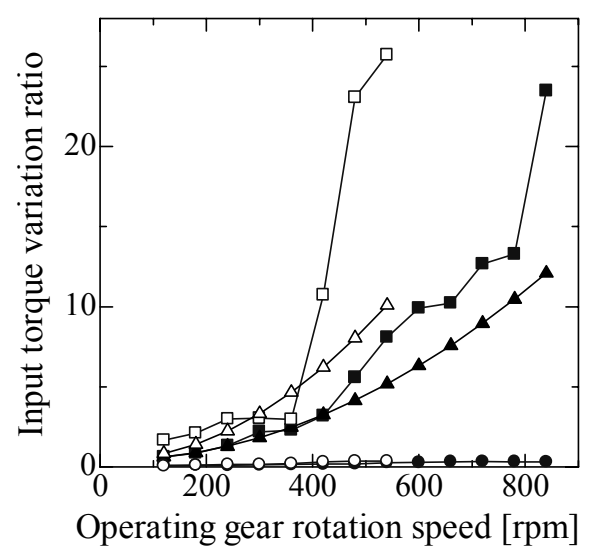

(a) $T_{l}=9.8 \mathrm{~N}-\mathrm{m}$

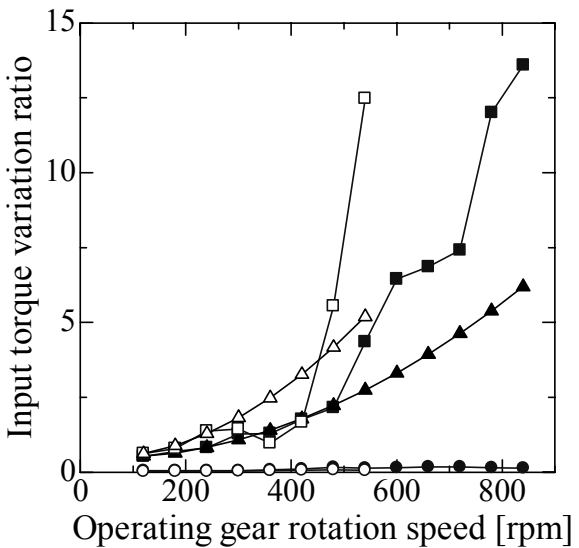

(b) $T_{l}=19.6 \mathrm{~N}-\mathrm{m}$

Fig. 8 Input torque variation ratios versus the operating rotation speeds for the four test gears

- Experimental results for elliptical gear I $\square-$ Experimental results of elliptical gear II

$\_$Calculation results for elliptical gear I $\neg$ Calculation results for elliptical gear II

- Experimental results for circular gear I —- Experimental results for circular gear II

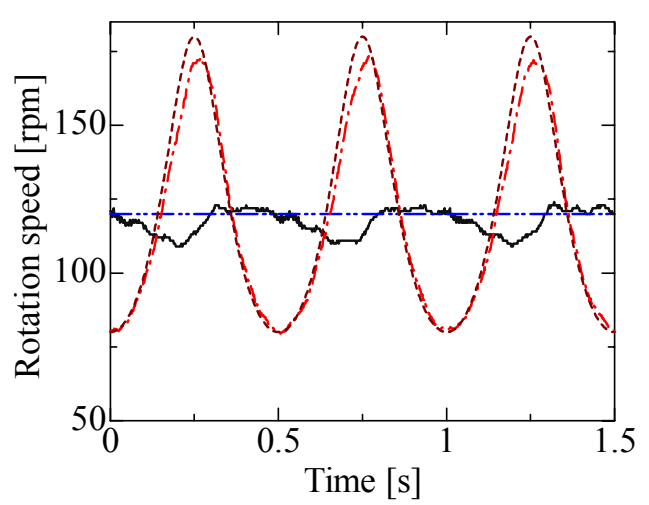

(a) $n=120 \mathrm{rpm}$

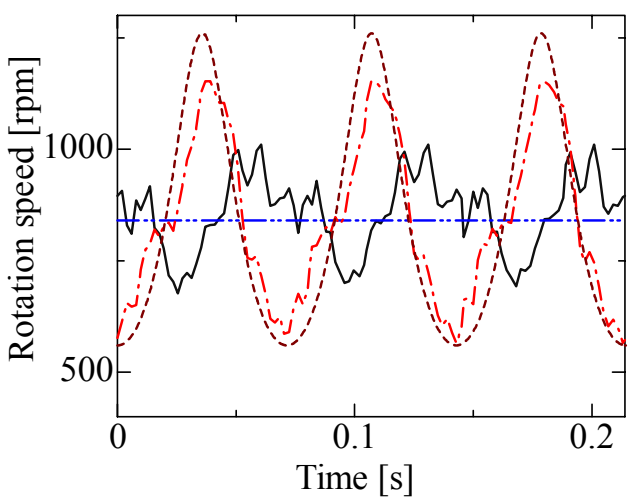

(b) $n=840 \mathrm{rpm}$

Fig. 9 Calculation and experimental results of rotation speed variation waveforms for Elliptical gear I, $T_{l}=9.8 \mathrm{~N}-\mathrm{m}$

_ Experimental input rotation speed --.- Experimental output rotation speed

-.--Calculated input rotation speed -----Calculated output rotation speed 
measured at the same time. The instantaneous AVRs of each time point of the calculated and experimental rotation speed variation waveforms are made consistent to make sure that the calculated and experimental rotation speed variation waveforms are at the same time.

As shown in Fig. 9, even at the operating gear rotation speed of $120 \mathrm{rpm}$, the experimental input rotation speed of elliptical gear varies periodically, with the variation frequency equal to the variation frequency of motion variation of elliptical gear. Recalling Fig. 2, it can be found that close to the angular position of maximum AAR, the experimental input rotation speed gets down to the bottom, while close to the angular position of minimum AAR the experimental input rotation speed rises to the top. The main reason for these is considered to be the response of the three-phase asynchronous motor toward the variation of dynamic input torque of elliptical gear. The largest inertial torque is yielded at the angular position of maximum AAR, while the smallest inertial torque is yielded at the angular position of minimum AAR. Since the experimental input rotation speed varies, the experimental output rotation speed also varies accordingly and goes away from the calculated ones which are the desired output rotation speeds. When the gear rotation speed changes from $120 \mathrm{rpm}$ to $840 \mathrm{rpm}$, the ratio of the maximum difference between the experimental output rotation speed and the calculated one to the operating gear rotation speed increases from $5 \%$ to $12.9 \%$ for elliptical gear I under load torque $9.8 \mathrm{~N}-\mathrm{m}$.

\subsection{Experimental results of the CRSTS of elliptical gear}

Figure 10 shows the comparison of the calculation and experimental results of the CRSTSs of elliptical gears versus load torques. The CRSTS of elliptical gear can be obtained by measuring the operating gear rotation speed when the measured tooth root stress on the tooth with the minimum AAR of elliptical gear becomes zero. As can be found in Fig. 10, as increasing the load torque, both the calculated and experimental CRSTSs of elliptical gears become higher, which shows that tooth separation of elliptical gear is inhibited by heavy load. And both the calculated and experimental CRSTSs of elliptical gear II are lower than that of elliptical gear I under the same load torque because of its larger AAR, i.e. inertial torque than elliptical gear I. The experimental CRSTS of elliptical gears is almost equal to the calculated one, which is considered as that at the operating gear rotation speed lower than the CRSTS of elliptical gear, the dynamic vibration is not so strong and the difference of the experimental input torque and calculated input torque is small. Therefore, one can estimate roughly the dynamic CRSTS of elliptical gear from the calculation.

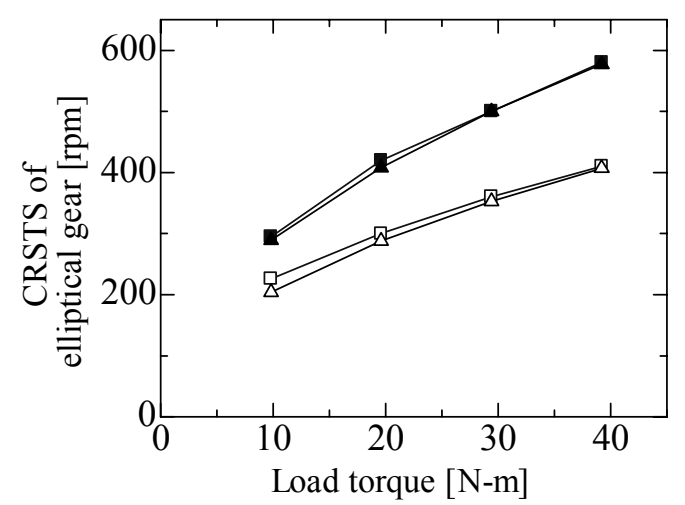

Fig. 10 Calculation and experimental results of the CRSTSs of elliptical gears

- Experimental results for elliptical gear I $\longrightarrow$-Experimental results of elliptical gear II

$\triangle$ Calculation results for elliptical gear I $\rightarrow$ Calculation results for elliptical gear II 


\subsection{Experimental results of tooth root stress}

Dynamic tooth root stress indicates the dynamic load between the meshing teeth. In this study, the tooth root stress of the non-mesh-side of the driven gear is measured by running test and used to illuminate the dynamic load of meshing teeth. Figure 11 shows examples of the experimental tooth root stress waveforms of the tooth No.14 and No.24 of elliptical gear I for $9.8 \mathrm{~N}-\mathrm{m}$, which are of the maximum and minimum AAR respectively. The pitch signal in Fig. 11 is of the same cycle as the tooth mesh cycle. As it can be found in Fig. 11, the experimental tooth root stress of different tooth of elliptical gear is different. The experimental tooth root stress of tooth No.14 increases along with the increase of operating gear rotation speed, however, that of tooth No.24 decreases.

In this study the experimental dynamic load variation ratio of elliptical gear, which is defined as the ratio of the maximum dynamic tooth root stress of elliptical gear at operating gear rotation speeds to that of corresponding circular gear at $120 \mathrm{rpm}$ which is the lowest operating gear rotation speed of the experiment, is used to describe the experimental dynamic load variation of elliptical gear with respect to operating gear rotation speed. Figure 12 shows the experimental dynamic load variation ratios at different angular position of elliptical gears versus operating gear rotation speed for load torque $9.8 \mathrm{~N}-\mathrm{m}$. As found in Fig. 12, the experimental dynamic load variation ratios of tooth No. 14 of elliptical gear I and tooth No.5 of elliptical gear II increase along with the increase of operating gear rotation speed, while those of tooth No. 24 of elliptical gear I and tooth No.16 of elliptical gear II decrease due to the inertial torques of elliptical gears. The experimental dynamic load variation ratios of tooth No. 14 of elliptical gear I and tooth No.5 of elliptical gear II get about 10 at $840 \mathrm{rpm}$ and at $540 \mathrm{rpm}$ respectively, while those of tooth No.24 of elliptical gear I and tooth No.16 of elliptical gear II get about -3 at $840 \mathrm{rpm}$ and -5 at $540 \mathrm{rpm}$ respectively. For other teeth of elliptical gear I and elliptical gear II, the curves of the experimental dynamic load variation ratios are probably between the curves of the tooth No.14 and tooth No.24 of elliptical gear I and the curves of tooth No.5 and tooth No.16 of elliptical gear II respectively. Comparing Fig. 12 with Fig. 6, it can be found that along with operating gear rotation speed the trends of the calculated and experimental dynamic load variation ratios of elliptical gears agree with each other. However, due to tooth separation and tooth impact, the experimental dynamic load variation ratios of elliptical gears show differences from the calculated ones.

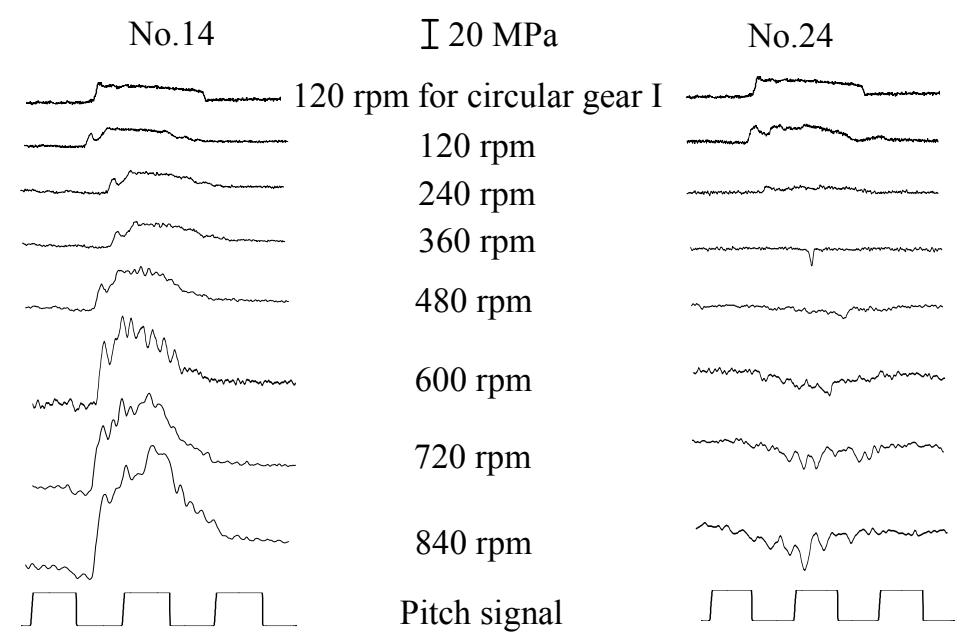

Fig. 11 Experimental tooth root stress waveforms of Elliptical gear I, for $T_{l}=9.8 \mathrm{~N}-\mathrm{m}$ 


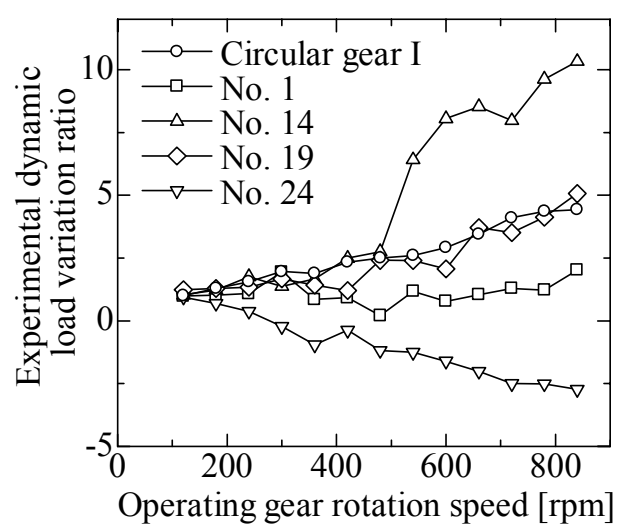

(a) Elliptical gear I

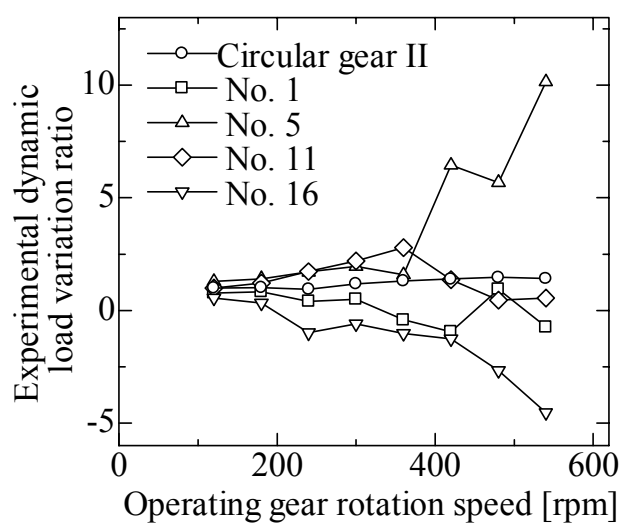

(b) Elliptical gear II

Fig. 12 Experimental dynamic load variation ratios of various teeth of elliptical gears, $T_{l}=9.8 \mathrm{~N}-\mathrm{m}$

\section{Conclusions}

Due to the non-uniform rotation of elliptical gear, the dynamic characteristics of elliptical gears demonstrate particular characteristics compared with circular gears.

1. Both input and output torques of elliptical gear vary periodically according to the non-uniform rotation of elliptical gear. The torque variation ratio of elliptical gear becomes much larger than that of circular gear along with the increase of operating gear rotation speed due to the inertial torque of elliptical gear. And the torque variation ratio of double elliptical gear is even larger than that of single elliptical gear because of the larger motion variation of double elliptical gear. As a result of the appearance of tooth impact, the experimental torque variations of elliptical gears are much larger than the calculated ones over the CRSTS of elliptical gears.

2. The experimental input rotation speed varies according to the variation of input torque. Since the experimental input rotation speed varies, the experimental output rotation speed of elliptical gear also varies and goes away from the calculated one. The maximum difference between the experimental output rotation speed and the calculated one of elliptical gear becomes larger along with the increase of operating gear rotation speed.

3. There is a CRSTS for a certain elliptical gear apparatus. Over the CRSTS of elliptical gear, tooth separation and non-mesh-side and mesh-side tooth impact occur, leading to the increase of dynamic load. When higher load torque is set, the CRSTS of elliptical gear gets higher, which indicates that tooth separation of elliptical gear is inhibited by heavy load. Between single elliptical gear and double elliptical gear, the CRSTS of double elliptical gear is larger due to its larger motion variation. The experimental CRSTS of elliptical gear is almost equal to the calculated one.

4. The tooth root stress of elliptical gear at different angular position is quite different from each other. Along with the increase of operating gear rotation speed in this study, the dynamic load variation ratio of elliptical gear at the tooth with the maximum AAR gets several times larger, while it decreases and becomes negative at the tooth with the minimum AAR. For other teeth of elliptical gear, the dynamic load variation ratios are probably between the above two values. The trends of the calculated and experimental dynamic load variation ratios of elliptical gears agree with each other. However, due to tooth separation and tooth impact, the experimental dynamic load variation ratios of elliptical gears show differences from the calculated ones.

The agreement of the calculation and experimental results proves the validity of this study. 


\section{References}

(1) Anonymous, Elliptical Gears Are Back and More Versatile Than Ever, Prod. Eng., Vol. 43, (August 1972), pp. 34-38.

(2) F. Freudenstein, C.K. Chen, Variable-Ratio Chain Drives with Noncircular Sprockets and Minimum Slack-Theory and Application, ASME J. Mech. Des., Vol. 113, No. 3 (1991), pp. 253-262.

(3) H. Katori, K. Yokogawa, T. Hayashi, a Simplified Synthetic Design Method of Pitch Curves Based on Motion Specifications for Noncircular Gears, Trans. Jpn. Soc. Mech. Eng., Vol. 60, No. 570 (1994), pp. 668-674 (in Japanese).

(4) S.H. Tong, C.H. Yang, Generation of Identical Noncircular Pitch Curves, ASME J. Mech. Des., Vol. 120, No. 2 (1998), pp. 337-341.

(5) G. Figliolini, J. Angeles, the Synthesis of Elliptical Gears Generated by Shaper-Cutter, ASME J. Mech. Des., Vol. 125, No. 4 (2003), pp. 793-801.

(6) G. Figliolini, J. Angeles, Synthesis of the Base Curves for N-Lobed Elliptical Gears, ASME J. Mech. Des., Vol. 127, No. 5 (2005), pp. 997-1005.

(7) A. Arakawa, T. Emura, Contact Ratio of Noncircular Gears, Trans. Jpn. Soc. Mech. Eng., Vol. 61, No. 585 (1995), pp. 2093-2099 (in Japanese).

(8) S.L. Chang, C.B. Tsay, L.I. Wu, Mathematical Model and Undercutting Analysis of Elliptical Gears Generated by Rack Cutters, Mech. Mach. Theory, Vol. 31, No. 7 (1996), pp. 879-890.

(9) B.W. Bair, Computer Aided Design of Non-Standard Elliptical Gear Drives, Proc. Instn. Mech. Egnrs., Part C: J Mechanical Engineering Science, Vol. 216, No.4 (2001), pp. 473-483.

(10) B.W. Bair, Computerized Tooth Profile Generation of Elliptical Gears Manufactured by Shaper Cutters, J. Mater. Process. Tech., Vol. 122, No. 2-3 (2002), pp. 139-147.

(11) B.W. Bair, Computer Aided Design of Elliptical Gears with Circular-Arc Teeth, Mech. Mach. Theory, Vol. 39, No.2 (2004), pp. 153-168.

(12) C.F. Chen, C.B. Tsay, Computerized Tooth Profile Generation and Analysis of Characteristics of Elliptical Gears with Circular-Arc Teeth, J. Mater. Process. Tech., Vol. 148, No.2 (2004), pp. 226-234.

(13) B.W. Bair, C.F. Chen, S.F. Chen, C.Y. Chou, Mathematical Model and Characteristic Analysis of Elliptical Gears Manufactured by Circular-arc Shaper Cutters, ASME, J. Mech. Des., Vol. 129, No. 2 (2007), pp. 210-217.

(14) F.L. Litvin, I. Gonzalez-Perez, K. Yukishima, A. Fuentes, K. Hayasaka, Generation of Planar and Helical Elliptical Gears by Application of Rack-Cutter, Hob and Shaper, Comput. Methods Appl. Mech. Engrg., Vol. 196, No. 41-44 (2007), pp. 4321-4336.

(15) B.W. Bair, Tooth Profile Generation and Analysis of Crowned Elliptical Gears, ASME, J. Mech. Des., Vol. 131, No. 7 (2009), pp. 074503-1-074503-6.

(16) T. Emura, A. Arakawa, a New Steering Mechanism Using Noncircular Gears, JSME Int. J. Ser. II, Vol. 35, No. 4 (1992), pp. 604-610.

(17) J-Y Liu, S-L Chang, D Mundo, Study on the Use of a Non-Circular Gear Train for the Generation of Figure-8 Patterns, Proc. Instn. Mech. Egnrs., Part C: J Mechanical Engineering Science, Vol. 220, No. 8 (2006), pp. 1229-1236.

(18) H. Katori, the Design, Manufacture and Application of Non-circular Gears, Tokyo, Nikkan Industrial Press, (2001), Chap.7, pp.141-156 (in Japanese).

(19) T. Aida, Y. Terauchi, on the Bending Stress of Spur Gear (3rd Report, on the Calculation Results of Bending Stress of Gear Tooth), Trans. Jpn. Soc. Mech. Eng., Vol. 27, No. 178 (1961), pp. 868-876 (in Japanese). 